\title{
Endometrial cancer-how many patients could benefit from sentinel lymph node dissection?
}

\author{
Sarah Brugger ${ }^{1 *}$, Moritz Hamann ${ }^{1}$, Marc Mosner ${ }^{1}$, Michaela Beer ${ }^{2}$, Michael Braun ${ }^{1}$ and Martin Pölcher ${ }^{1}$
}

\begin{abstract}
Background: Sentinel lymph node dissection (SLND) may reduce morbidity in patients with endometrial cancer. The objective of this study is to estimate how many systematic lymph node dissections (LND) can be spared with an implementation of a SLN-procedure.

Methods: Retrospective, single-center study, SLND according to NCCN-Guidelines.

Results: In 109 patients of 154 consecutive patients, SLND was performed. The detection rate was 61\% on both sides and $86 \%$ on at least one side. Classification of uterine risk factors is as follows: low risk 53 , intermediate risk 25 , high-intermediate risk 13, and high-risk 18. Stage IIIC: 0, 3, 7, 11, respectively. Under the assumption that 56 patients with "higher than low risk" factors would be treated by systematic LND, we spared 26 pelvic and paraaortic LND. After failures of SLN detection, unilateral pelvic LND was performed in 15 patients. Patients with "higher than low risk" factors and node-negative SLN are candidates for a randomized study to prove safety and efficacy. Only every third patient in our study met these criteria.

Conclusions: In a cohort of patients with "higher than low risk" endometrial cancer, the implementation of SLND nearly divided the number of radical lymph node dissections in half. Further studies are required to define the best modalities for SLND.
\end{abstract}

Keywords: Risk groups, Ultrastaging, Detection rate

\section{Background}

Endometrial cancer is the most common carcinoma of the female genital tract with over 300,000 new cases diagnosed each year worldwide [1]. Comprehensive surgical staging in endometrial cancer, i.e., hysterectomy, bilateral salpingo-oophorectomy, and lymph node assessment by pelvic and paraaortic lymph node dissection (LND), has been controversial for many years. Patients with lymph node metastasis have significant lower median survival rates compared to patients with a tumor confined to the uterus [2]. The finding of nodal involvement therefore is one of the most important diagnostic factors to initiate adjuvant treatment.

\footnotetext{
* Correspondence: sarah.brugger@swmbrk.de

${ }^{1}$ Rotkreuzklinikum München, Frauenklinik Taxisstraße 3, 80637 München, Germany

Full list of author information is available at the end of the article
}

The probability of lymph node metastases can be determined by tumor characteristics like histological subtype, grading, lymph vessel space invasion, and myometrial invasion (Table 1). A risk-adopted management of LND according to current consensus statements provides a simple hysterectomy without lymph node assessment in patients with uterine low-risk factors and a systematic lymph node dissection in patients with high-risk factors [3]. In patients with intermediate-risk factors or highintermediate-risk factors, the management is weighting of risks, and the best practice is not yet established.

In gynecologic cancers, LND can cause severe long term-morbidity, especially lymph edema of the legs and should be omitted whenever appropriate [4, 5]. Preoperative imaging does not correspond well with postoperative findings [6]. Therefore, surgical staging remains the gold standard in the assessment of lymph node status. Systematic lymph node dissection was a dogma of

(C) The Author(s). 2018 Open Access This article is distributed under the terms of the Creative Commons Attribution 4.0 International License (http://creativecommons.org/licenses/by/4.0/), which permits unrestricted use, distribution, and 
Table 1 Risk groups according to ESMO-ESGO-ESTRO Consensus Conference [3]

\begin{tabular}{lllll}
\hline & Grading & Histological type & Stage & LVSI \\
\hline Low risk & $\mathrm{G} 1, \mathrm{G} 2$ & Endometrioid & IA & Negative \\
Intermediate risk & $\mathrm{G} 1, \mathrm{G} 2$ & Endometrioid & IA & Nesitive \\
& $\mathrm{G} 1, \mathrm{G} 2$ & Endometrioid & IB & Negative \\
High-intermediate risk & $\mathrm{G} 3$ & Endometrioid & IA & Positive \\
High risk & $\mathrm{G} 3$ & Endometrioid & IA & Positive \\
& $\mathrm{G} 1, \mathrm{G} 2$ & Endometrioid & IB & Negative/positive \\
& $\mathrm{G} 3$ & Endometrioid & IA/B & Negative/positive \\
& $\mathrm{G} 3$ & & Negative/positive \\
\hline
\end{tabular}

Stages IA myometrial invasion $<50 \%$, IB myometrial invasion $\geq 50 \%$; stage /I cervical involvement; LVSI lymphovascular space invasion

cancer surgery in the past century. Its role as a therapeutic procedure has become controversial in different cancer types, e.g., breast and ovarian cancer [7-10], especially when adjuvant treatment follows lymph node surgery.

Sentinel lymph node dissection (SLND) can serve as a useful tool whenever reduction of morbidity can be achieved without lacking the information concerning nodal involvement, adjuvant treatment, and prognosis. Sentinel lymph node mapping as introduced by AbuRustum means removal of any colored or suspicious lymph nodes. This might be a common ground between no lymph node assessment and radical pelvic and paraaortic LND [11, 12].

To prove efficacy and safety of this approach, a prospective and randomized trial would be appropriate. Two randomized studies in endometrial cancer could not show a benefit for patients who have undergone systematic LND in recurrence-free survival (RFS) or overall survival (OS) compared with patients without LND [13, 14]. Three major concerns have been raised over these data. Firstly, a great proportion of patients with uterine low-risk factors may have led to the concealment of a survival difference in patients who were at higher risk. Secondly, the lymph node assessment like sampling and bulky node dissection in patients randomized for omitting lymph node assessment may have caused a crossover effect. And lastly, the lack of standardized adjuvant treatment regimens led to an unequal distribution of patients who had adjuvant radiotherapy or chemotherapy [15].

The aim of this study was to incorporate a sentinel lymph node (SLN) procedure with a special focus on the proportion of patients who could benefit most from this procedure: how many patients have a negative sentinel lymph node and are at a higher risk than low risk, i.e., intermediate risk, high-intermediate risk, or high risk? In this group, a systematic LND can be omitted by the implementation of a reliable SLN procedure. To prove safety and efficacy within a clinical trial, this "higher than low risk" group would serve as potential target population for a phase 3 protocol. We tried to determine the feasibility of a study with regard to the experience of the former studies.

\section{Methods}

In this retrospective single-center study, lymph node assessment by SLND was offered in all assumed stage I cancers and registered in a prospectively maintained database. Inclusion criteria for this study were histologically confirmed endometrial cancer by dilation and curettage $(\mathrm{D} / \mathrm{C})$ and disease confined to the uterine corpus in transvaginal ultrasound imaging (stage I disease). Patients had to be deemed fit for major abdominal surgery and potential pelvic and paraaortic lymph node dissection.

Exclusion criteria were known allergic reaction to patent blue dye, node positive, or locally advanced disease in previous imaging. Computer tomography or magnetic resonance imaging was not mandatory; however, if patients presented to our clinic with preoperative imaging, they were excluded if locally advanced disease or lymph node metastasis was suspected. One surgeon performed the first 50 SLND and two further surgeons the SLND of the entire population. Informed consent was obtained from all individual participants included in the study.

A total of 4-ml patent blue dye was injected into the cervix at 3 and 9 o'clock (1-mm and $1-\mathrm{cm}$ depth, $1 \mathrm{ml}$ respectively) at the beginning of surgery according to the National Comprehensive Cancer Network (NCCN) guidelines [16]. Detection and removal of sentinel lymph nodes was then performed for each side. If detection of a SLN failed, no definitive proceeding was stated. This means that pelvic and paraaortic LND, pelvic LND on one or both sides, or no further lymph node assessment was performed at the discretion of the surgeon. If detection of sentinel lymph node failed, pelvic and paraaortic LND, pelvic LND on one or both sides, or no further 
lymph node assessment was performed at the discretion of the surgeon. In patients with short history of postmenopausal vaginal bleeding, inconspicuous ultrasound findings, good differentiation after $\mathrm{D} / \mathrm{C}$, lack of lymphovascular space invasion, or atypical hyperplasia with transition into a well differentiated carcinoma, a great probability of a low-risk situation was to assume. In these cases, pelvic LND was omitted even if the SLN was not detected. LND was performed within a second operation if the final histopathological reports showed uterine risk factors "higher than low risk". In patient with supposed uterine risk factors "higher than low risk" (e.g., suspected $\mathrm{MI}>50 \%$ in ultrasound diagnostic, G3/ type2/LVSI in the $\mathrm{D} / \mathrm{C}$ specimen), consecutive lymph node assessment was performed within the same operation.

For better detection of low volume metastasis, ultrastaging of all SLN was performed by additional serial sectioning and immunohistochemical staining. Definition of lymph node micrometastasis was $0.2-2.0 \mathrm{~mm}$ and ITC up to $0.2 \mathrm{~mm}$.

\section{Results}

Between February 2013 and February 2017, a total of 154 patients with endometrial cancer were treated by surgery at our institution. A total of 45 patients (29\%) were ineligible for SLND: Advanced disease was suspected in 16 patients (local advanced disease (1)/lymph node metastases (8)/distant metastases (7)). General or geriatric assessment deemed 10 patients with a median age of 85 years to be unsuitable for LND. In 9 patients, previous hysterectomy showed unexpected carcinoma; therefore, the opportunity for sentinel mapping was missed. Refusing written informed consent, 10 patients were excluded, the main reason being due to having concerns about lymph node surgery.

SLND was performed in 109 patients. Patient characteristics and risk assessments are shown in Table 2. Overall, node-positive disease was detected in 19\% ( $n=$ $21)$. In the low-risk group, no lymph node metastases were detected; in the intermediate $12 \%(n=3)$; in the high-intermediate-risk group $54 \%(n=7)$; and in the high-risk group $61 \%(n=11)$. A change of the grading score after hysterectomy-in contrast to the tumor characteristics available by curettage specimens-occurred in $14 \%$ of patients $(n=15)$.

The detection rate of the sentinel node was $61 \%(n=$ $67)$ on both sides and $86 \%(n=94)$ on at least one side. The detection on the right pelvic side succeeded in 86 patients, on the left pelvic side in 74 patients. On average, 2.4 lymph nodes were removed on the right side and 2.0 lymph nodes on the left side. In 4 patients (3. $7 \%)$ with "higher than low risk" tumors, the lymph node metastases were detected by ultrastaging of the sentinel
Table 2 Patient characteristics of 109 SLND

\begin{tabular}{|c|c|c|c|}
\hline Age, years (range) & Median & 63.5 & $(28-89)$ \\
\hline \multirow[t]{3}{*}{ Grading, uterus-specimen } & G1 & 57 & $52 \%$ \\
\hline & $\mathrm{G} 2$ & 30 & $28 \%$ \\
\hline & G3 & 22 & $20 \%$ \\
\hline \multicolumn{4}{|l|}{ Histopathological type } \\
\hline Type I & & 99 & $91 \%$ \\
\hline \multirow[t]{4}{*}{ Type II } & & 10 & $9 \%$ \\
\hline & Serous & & 6 \\
\hline & Clear cell & & 1 \\
\hline & Carcinosarcoma & & 3 \\
\hline \multirow[t]{4}{*}{ Uterine risk factors } & Low risk & 53 & $49 \%$ \\
\hline & Intermediate risk & 25 & $23 \%$ \\
\hline & High-intermediate risk & 13 & $12 \%$ \\
\hline & High risk & 18 & $17 \%$ \\
\hline \multirow[t]{4}{*}{ Nodal involvement } & Low risk & 0 & $0 \%$ \\
\hline & Intermediate risk & 3 & $12 \%$ \\
\hline & High-intermediate risk & 7 & $54 \%$ \\
\hline & High risk & 11 & $61 \%$ \\
\hline \multirow[t]{7}{*}{ Final FIGO stage } & IA & 60 & $55 \%$ \\
\hline & $\mathrm{IB}$ & 23 & $21 \%$ \\
\hline & $\|$ & 5 & $5 \%$ \\
\hline & $\| I C 1$ & 10 & $9 \%$ \\
\hline & $\mathrm{IIC} 2$ & 5 & $5 \%$ \\
\hline & $\| \mathrm{A}$ & 1 & $1 \%$ \\
\hline & IVB & 5 & $5 \%$ \\
\hline
\end{tabular}

nodes. These patients may have been falsely classified as lymph node negative with conventional staining methods if a lymph node dissection would have been chosen. In addition, isolated tumor cells (ITCs) were found in 4 out of 94 patients (4.3\%).

A risk-adopted management would have caused pelvic and paraaortic LND in all patients "higher than low risk" $(n=56)$. In this study, we performed 109 SLN procedures. We performed 30 pelvic and paraaortic LND and 15 pelvic unilateral LNDs (8 of them were low-risk patients). In 65 patients, no further lymph node dissection was performed. In 38 cases, LND was performed simultaneously and in 7 cases within a second operation. Management after failure of sentinel detection is shown in Table 3.

After successful detection of SLN on both sides, 16 pelvic and paraaortic LND identified 12 node-positive and only four node-negative patients. The lymph node status was correctly predicted by SLND in all 16 cases. In this small subgroup, the false-negative value was $0 \%$.

In one patient with high-intermediate risk, we found isolated paraaortic lymph node metastases after failure 
Table 3 Management of lymph node dissection after injection of blue dye for sentinel lymph node dissection

\begin{tabular}{|c|c|c|c|c|c|c|c|c|c|c|}
\hline & \multicolumn{2}{|c|}{$\begin{array}{l}\text { Low risk } \\
n\end{array}$} & \multicolumn{2}{|c|}{$\begin{array}{l}\text { Intermediate risk } \\
n\end{array}$} & \multicolumn{2}{|c|}{$\begin{array}{l}\text { High-intermediate risk } \\
n\end{array}$} & \multicolumn{2}{|c|}{$\begin{array}{l}\text { High risk } \\
n\end{array}$} & \multicolumn{2}{|c|}{ Sum } \\
\hline SLN detection failed on both sides & 7 & & 4 & & 0 & & 4 & & 15 & \\
\hline No further dissection & & 7 & & 1 & & & & 0 & & 8 \\
\hline Pelvic LND & & 0 & & 0 & & & & 0 & & 0 \\
\hline Pelvic and para-aortic LND & & 0 & & 3 & & & & 4 & & 7 \\
\hline SLN detection failed on one side & 13 & & 3 & & 5 & & 6 & & 27 & \\
\hline No further dissection & & 6 & & 0 & & 0 & & 2 & & 8 \\
\hline Pelvic LND & & 7 & & 2 & & 3 & & 0 & & 12 \\
\hline Pelvic and para-aortic LND & & 0 & & 1 & & 2 & & 4 & & 7 \\
\hline SLN detection successful on both sides & 33 & & 18 & & 8 & & 8 & & 67 & \\
\hline No further dissection & & 33 & & 11 & & 6 & & 1 & & 51 \\
\hline Pelvic LND & & 0 & & 0 & & 0 & & 0 & & 0 \\
\hline Pelvic and para-aortic LND & & 0 & & 7 & & 2 & & 7 & & 16 \\
\hline Sum & 53 & & 25 & & 13 & & 18 & & & 109 \\
\hline
\end{tabular}

SLN sentinel lymph node, LND lymph node dissection

of sentinel detection on one pelvic side; 3 of 63 lymph nodes were involved. Only one SLN was found in the paracaval region and was negative.

Only one patient in the "higher than low risk" group with stage IB G1 had no lymph node assessment on both sides due to having no detection of a SLN on one side and refusing a second operation for systematic LND.

Of 109 patients, 56 tumors were "higher than low risk". In 34 patients of this cohort, no lymph node involvement was found. These patients represent the group who may benefit most from a sentinel lymph node dissection as systematic dissection can be omitted.

In two patients, a conversion from laparoscopy to open surgery occurred for intraoperative bleeding or unexpected disseminated peritoneal carcinomatosis. In one patient, there was a thermic lesion at the appendix with consecutive appendectomy. In another patient, an ileum perforation occurred with consecutive bowel resection after laparoscopic LND.

Postoperative complications within 28 days included the following: transitory weakness/numbness of the legs $(n=3)$, vaginal bleeding or hematoma of the vaginal vault $(n=3)$, trocar hernia with consecutive surgery $(n=$ $2)$, surgical site infection after laparotomy $(n=5)$, transfusion for postoperative anemia $(n=2)$, and mechanically ileus $(n=1)$. There were no significant differences between patients with hysterectomy and SLND compared to those with pelvic or pelvic and paraaortic LND.

\section{Discussion}

The current concept of lymph node assessment follows a risk-adopted algorithm. It suggests a systematic LND in patients with endometrial cancer and uterine highrisk factors. In these patients, the incidence of lymph node involvement is high, as seen in this trial with $61 \%$ node-positive patients. For patients with low-risk factors, lymph node assessment may be omitted. In this study, neither lymph node metastases nor micrometastases (MM) were found after SLND in 53 lowrisk patients-even after a thorough examination, so called ultrastaging.

In patients with intermediate and high-intermediaterisk factors, the significance of LND is not clear [3]. The proportion of these two groups, however, was $35 \%$ of the entire patient cohort, and we found lymph node metastases in $12 \%$ of the intermediate-risk group and $54 \%$ in the high-intermediate-risk group. This indicates that lymph node assessment is potentially meaningful for these patient groups [17].

A weakness of the current risk-adopted lymph node management is that the proper risk group cannot safely be determined at the time of surgery: depth of invasion, grading, lymph vessel invasion, and cervical involvement are factors needed for the indication of a LND. Intraoperative pathology is not always a reliable method for determining these factors [18-21]. In this study, grading differed in $14 \%$ comparing curettage specimen with hysterectomy specimen. Therefore, patients may suffer a second operation if risk factors change after hysterectomy and a systematic LND is recommended.

In contrast, the $S L N$ procedure is used in all patients, regardless of special risk factors. Detection can only take place before removing the uterus because blue dye tracer colors lymph vessels and nodes only temporarily. Therefore, a high number of rather optional SLND in low-risk patients has to be accepted. The concept of SLND therefore introduces a totally different approach. The most challenging issue in SLND is the surgical management 
in case of non-detection of a SLN. As a midline organ, uterine lymphatic vessels drain on both pelvic sides and therefore detection of SLN on both sides is recommended. The NCCN-algorithm provides for systematic pelvic LND on the side, where the SLN is not detected. This enables a one-stop strategy, and therefore, a second operation is not intended.

We did both: immediate consecutive LND in cases of non-detection of the SLN, especially in patients with known risk factors "higher than low risk" and secondary operation with LND, e.g., in patients with a positive ultrastaged SLN. To detect $21 \%$ node-positive patients, we performed lymph node assessment with at least one pelvic LND in $41 \%$ of the entire cohort of SLN patients. In 30 patients, a pelvic and paraaortic LND was performed. Assumed that pelvic and paraaortic LND would be reasonable for all 56 patients higher than low risk, we nearly divided the number of radical lymph node dissections in half.

Reconsidering the main critical comments on former lymph node dissection trials, a study with a high proportion of low-risk patients might not be suitable to show the equivalence of different surgical lymph node assessments [15]. Therefore, a trial should only include patients of the "higher than low-risk" group. A comparison of both strategies, "risk adopted" vs. "SLN mapping," would be one protocol option. Another option could be a study providing for LND in cases of positive SLN and in cases of negative SLN with a randomization into systematic LND or observation. As shown in this study, a feasible randomized trial to measure safety and outcome of SLND versus LND requires an exceedingly large number of patients as only approximately every third patient undergoing SLND would be eligible for randomization.

A study comparing outcome of surgical procedures should predefine adjuvant treatment. Regimens for patients with higher risk of recurrence or node-positive disease with either chemotherapy, radiation therapy, or both are being investigated in several clinical trials (PORTEC 1, GOG 99, PORTEC-2, GOG 122, GOG177, RTOG 0763) [22-26]. Recently, first results of a phase 3 trial (PORTEC-3) addressing the benefit of adjuvant chemotherapy during and after radiotherapy versus pelvic radiotherapy alone for women with high risk and advanced endometrial cancer were reported. No difference was found in progression-free survival (PFS) [27]. However, in the subgroup of all stage III patients including those with nodal involvement, there was a significant benefit in the 5-year PFS for the combined regimen $(69.3$ vs. $58.0 \%$ [95\% CI $0.45-0.97, p=0.032]$ ). This study can help to determine a standardized adjuvant treatment within a protocol.

To date, there are no prospective studies demonstrating a survival benefit for a systematic pelvic and paraaortic LND. It is questionable whether a clinical study could find a difference between two types of lymph node assessments, assuming there is one. The risk of missed paraaortic metastases within the SLN procedure could be offset by the higher detection rate of stage III disease by ultrastaging. Hence, a protocol for a largescale validation trial of lymph node management is a challenge.

Technically, the implementation succeeded without any problems: We instantly achieved a detection rate that is exactly on par with the literature [28-30]. Several studies were reported to improve detection rates with different tracers or injection sites. A new technique utilizes indocyanin-green (ICG) and the fluorescence signal can be detected with a special infrared sensor [31, 32]. Recent studies point out that ICG might develop as a new standard in detection of sentinel lymph nodes due to the fact it shows better detection rates, especially in patients with a high body mass index [33]. Laparoscopic injection into the uterine fundus has not been established, as detection rates were low. The main criticism of the cervical injection site is a possible lack of representative mapping in the paraaortic area. In a retrospective analysis, a survival benefit for patients with paraaortic lymph node dissection in endometrial cancer was described, indicating that nodal involvement in this area is clinically meaningful [34]. In one patient, after failure of SLN detection, we found a paraaortic lymph node metastasis without pelvic positive nodes. These isolated paraaortic metastases are rare with rates ranging from 1 to $1.5 \%$ [35-37].

Ultrastaging of sentinel lymph nodes leads to a higher detection rate of low-volume lymph node metastases [38]. In our study, metastases were detected by ultrastaging in $4 \%$ of the cases which were not detected by conventional histology. This number is even lower than published in literature with upstaging as a result of ultrastaging in up to $20 \%$ [39-41], though the prognostic value of low-volume lymph node metastases and the best management of this finding are not yet established. Patients with ITC and MM received adjuvant chemotherapy more frequently and showed a better oncologic outcome when compared to patients with macrometastasis over a time period of 3 years $[40,42]$. Bezu et al. found a link between the occurrence of MM and higher recurrence rate of uterine cancers [43].

A prospective multicenter cohort study with indocyanine green in 340 patients identified 35 of 36 patients with lymph node metastases by SLN mapping and consecutive dissection, yielding a sensitivity of $97.2 \%$, a negative predictive value of $99.6 \%$ and a false-negative rate of 3\% [29]. In a meta-analysis which included 4915 women, Smith et al. concludes that sentinel mapping accurately predicts nodal status in women with 
endometrial cancer and may be considered an alternative standard of care in the staging of women with endometrial cancer [30]. Holloway et al. raise the question, whether SLND is the more precise method to target lymph node involvement [44].

Several limitations of this study should be emphasized. We did not perform LND after SLND in generally and therefore cannot report our false-negative rate. Preoperative imaging targeting myoinvasion or lymph node status was not standardized. Therefore, the assumption of stage I disease was made on different basic principals in the screening of 154 patients. Deep myometrial or cervical invasion in magnetic resonance imaging may have influenced decisions concerning lymph node management. No fixed algorithm after non-detection of sentinel nodes was provided; in some patients, we temporized the final histopathologic report to estimate final risk factors, for example in patients with supposed low-risk features.

A withdrawal of radical lymph node surgery was recently observed in breast and ovarian cancer [7, 10]. Therefore, several questions have to be addressed in the future of endometrial cancer treatment. It is not proven that systematic LND is mandatory in each case of positive SLN, especially when adjuvant treatments are scheduled. The role of lymph node status in order to guide postoperative management may be replaced at some point by other prognostic factors, e.g. CD171 [45]. Genetic profiling showed different subtypes after next generation sequencing in endometrial cancer. This may help to identify more adequate candidates for adjuvant treatment in terms of targeted therapies $[46,47]$.

\section{Conclusion}

Current surgical practices in endometrial cancer today range from no assessment of lymph nodes to comprehensive lymph node dissection. Both strategies are at high risk of over- and undertreatment. Sentinel lymph node dissection in endometrial cancer is a promising technique and, therefore, a step towards individualized treatment. Systematic pelvic and paraaortic LND can be minimized. However, safety data are lacking. A requested randomized trial poses a challenge in protocol design and patient recruitment.

\begin{abstract}
Abbreviations
D/C: Dilatation and curettage; ITC: Isolated tumor cells; LND: Lymph node dissection; MM: Micrometastases; NCCN: National Comprehensive Cancer Network; SLN: Sentinel lymph node; SLND: Sentinel lymph node dissection
\end{abstract}

\section{Availability of data and materials}

The datasets used and/or analyzed during the current study are available from the corresponding author on reasonable request.

\section{Authors' contributions}

$\mathrm{MM}, \mathrm{SB}$, and $\mathrm{MH}$ participated in collecting the patient data and analyzed and interpreted the patient data. $\mathrm{MBr}$ and MP were responsible for the outline of the research and data interpretation. MBe was in charge of the histopathology examination, including the process of ultrastaging. SB and MP were the major contributors in writing the manuscript. All authors read and approved the final manuscript.

\section{Ethics approval and consent to participate}

All patients gave written informed consent. The authors did a retrospective cohort study of patients treated. They did not seek ethical committee approval for their study, and provided the following justification for not having done so:

All data analyzed were collected as part of routine diagnosis and treatment. Patients were diagnosed and treated according to international guidelines and agreements.

\section{Competing interests}

The authors declare that they have no competing interests.

\section{Publisher's Note}

Springer Nature remains neutral with regard to jurisdictional claims in published maps and institutional affiliations.

\section{Author details}

${ }^{1}$ Rotkreuzklinikum München, Frauenklinik Taxisstraße 3, 80637 München, Germany. ${ }^{2}$ Pathologie Rotkreuzklinikum, Winthirstraße 11, 80639 München, Germany.

Received: 11 December 2017 Accepted: 2 May 2018

Published online: 17 May 2018

\section{References}

1. WHO. GLOBOCAN 2012: Estimated cancer incidence, mortality and prevalence worldwide in 2012. http://globocan.iarc.fr/Pages/fact_sheets_ population.aspx. Date last accessed 01 June 2017.

2. Zhou J, He ZY, Li FY, Sun JY, Lin HX, Wu SG, Chen QH. Prognostic value of lymph node ratio in stage IIIC epithelial ovarian cancer with node-positive in a SEER population-based study. Oncotarget. 2016;7:7952-9.

3. Colombo N, Creutzberg C, Amant F, Bosse T, Gonzalez-Martin A, Ledermann J, Marth C, Nout R, Querleu D, Mirza MR, Sessa C. ESMO-ESGO-ESTRO consensus conference on endometrial cancer: diagnosis, treatment and follow-up. Int J Gynecol Cancer. 2016;26:2-30.

4. Abu-Rustum NR, Alektiar K, lasonos A, Lev G, Sonoda Y, Aghajanian C, Chi DS, Barakat RR. The incidence of symptomatic lower-extremity lymphedema following treatment of uterine corpus malignancies: a 12-year experience at Memorial Sloan-Kettering Cancer Center. Gynecol Oncol. 2006;103:714-8.

5. Biglia N, Librino A, Ottino MC, Panuccio E, Daniele A, Chahin A. Lower limb lymphedema and neurological complications after lymphadenectomy for gynecological cancer. Int J Gynecol Cancer. 2015;25:521-5.

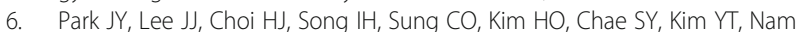
$\mathrm{JH}$. The value of preoperative positron emission tomography/computed tomography in node-negative endometrial cancer on magnetic resonance imaging. Ann Surg Oncol. 2017;24(8):2303-10. https://doi.org/10.1245/ s10434-017-5901-8. Epub 2017 May 26.

7. Bromham N, Schmidt-Hansen M, Astin M, Hasler E, Reed MW. Axillary treatment for operable primary breast cancer. Cochrane Database Syst Rev. 2017;1:CD004561.

8. Darling GE, Allen MS, Decker PA, Ballman K, Malthaner RA, Inculet RI, Jones DR, McKenna RJ, Landreneau RJ, Rusch WW, Putnam JB Jr. Randomized trial of mediastinal lymph node sampling versus complete lymphadenectomy during pulmonary resection in the patient with N0 or N1 (less than hilar) non-small cell carcinoma: results of the American College of Surgery Oncology Group Z0030 trial. J Thorac Cardiovasc Surg. 2011;141:662-70.

9. Leiter U, Stadler R, Mauch C, Hohenberger W, Brockmeyer N, Berking C, Sunderkotter C, Kaatz M, Schulte KW, Lehmann P, et al. Complete lymph node dissection versus no dissection in patients with sentinel lymph node biopsy positive melanoma (DeCOG-SLT): a multicentre, randomised, phase 3 trial. Lancet Oncol. 2016;17:757-67.

10. LION: Lymphadenectomy in ovarian neoplasms-A prospective randomized AGO study group led gynecologic cancer intergroup trial. http://abstracts. asco.org/199/AbstView_199_183691.html. 
11. Abu-Rustum NR. Update on sentinel node mapping in uterine cancer: 10year experience at Memorial Sloan-Kettering Cancer Center. J Obstet Gynaecol Res. 2014;40:327-34.

12. Barlin JN, Khoury-Collado F, Kim CH, Leitao MM Jr, Chi DS, Sonoda Y, Alektiar K, DeLair DF, Barakat RR, Abu-Rustum NR. The importance of applying a sentinel lymph node mapping algorithm in endometrial cancer staging: beyond removal of blue nodes. Gynecol Oncol. 2012;125:531-5.

13. Benedetti Panici P, Basile S, Maneschi F, Alberto Lissoni A, Signorelli M, Scambia G, Angioli R, Tateo S, Mangili G, Katsaros D, et al. Systematic pelvic lymphadenectomy vs. no lymphadenectomy in early-stage endometrial carcinoma: randomized clinical trial. J Natl Cancer Inst. 2008;100:1707-16.

14. Kitchener H, Swart AM, Qian Q, Amos C, Parmar MK. Efficacy of systematic pelvic lymphadenectomy in endometrial cancer (MRC ASTEC trial): a randomised study. Lancet. 2009;373:125-36.

15. Naumann RW. The role of lymphadenectomy in endometrial cancer: was the ASTEC trial doomed by design and are we destined to repeat that mistake? Gynecol Oncol. 2012;126:5-11.

16. NCCN-Clinical Practice guidelines in Oncology, Uterine Neoplams. https:// www.nccn.org/professionals/physician_gls/pdf/uterine.pdf.

17. Ouldamer L, Bendifallah S, Body G, Canlorbe G, Touboul C, Graesslin O, Raimond E, Collinet P, Coutant C, Lavoue V, et al. Call for surgical nodal staging in women with ESMO/ESGO/ESTRO high-intermediate risk endometrial cancer: a multicentre cohort analysis from the FRANCOGYN Study Group. Ann Surg Oncol. 2017;24:1660-6.

18. Case AS, Rocconi RP, Straughn JM Jr, Conner M, Novak L, Wang W, Huh WK. A prospective blinded evaluation of the accuracy of frozen section for the surgical management of endometrial cancer. Obstet Gynecol. 2006;108: 1375-9.

19. Egle D, Grissemann B, Zeimet AG, Muller-Holzner E, Marth C. Validation of intraoperative risk assessment on frozen section for surgical management of endometrial carcinoma. Gynecol Oncol. 2008;110:286-92.

20. Quinlivan JA, Petersen RW, Nicklin JL. Accuracy of frozen section for the operative management of endometrial cancer. BJOG. 2001;108:798-803.

21. Sethasathien P, Charoenkwan K, Siriaunkgul S. Accuracy of intraoperative gross examination of myometrial invasion in stage I-II endometrial cancer. Asian Pac J Cancer Prev. 2014;15:7061-4.

22. Creutzberg CL, Nout RA, Lybeert ML, Warlam-Rodenhuis CC, Jobsen J, Mens JW, Lutgens LC, Pras E, van de Poll-Franse LV, van Putten WL, Group PS. Fifteen-year radiotherapy outcomes of the randomized PORTEC-1 trial for endometrial carcinoma. Int J Radiat Oncol Biol Phys. 2011:81:e631-8.

23. Fleming GF, Brunetto VL, Cella D, Look KY, Reid GC, Munkarah AR, Kline R, Burger RA, Goodman A, Burks RT. Phase III trial of doxorubicin plus cisplatin with or without paclitaxel plus filgrastim in advanced endometrial carcinoma: a gynecologic oncology group study. J Clin Oncol. 2004;22: 2159-66.

24. Keys HM, Roberts JA, Brunetto VL, Zaino RJ, Spirtos NM, Bloss JD, Pearlman A, Maiman MA, Bell JG. A phase III trial of surgery with or without adjunctive external pelvic radiation therapy in intermediate risk endometrial adenocarcinoma: a Gynecologic Oncology Group study. Gynecol Oncol. 2004;92:744-51.

25. Nout RA, Smit VT, Putter H, Jurgenliemk-Schulz IM, Jobsen JJ, Lutgens LC, van der Steen-Banasik EM, Mens JW, Slot A, Kroese MC, et al. Vaginal brachytherapy versus pelvic external beam radiotherapy for patients with endometrial cancer of high-intermediate risk (PORTEC-2): an open-label, non-inferiority, randomised trial. Lancet. 2010;375:816-23.

26. Randall ME, Filiaci VL, Muss H, Spirtos NM, Mannel RS, Fowler J, Thigpen JT Benda JA. Randomized phase III trial of whole-abdominal irradiation versus doxorubicin and cisplatin chemotherapy in advanced endometrial carcinoma: a Gynecologic Oncology Group Study. J Clin Oncol. 2006;24:36-44

27. Final results of the international randomized PORTEC-3 trial of adjuvant chemotherapy and radiation therapy (RT) versus RT alone for women with high-risk endometrial cancer. http://abstracts.asco.org/199/AbstView_199_ 180760.html.

28. Kang S, Yoo HJ, Hwang JH, Lim MC, Seo SS, Park SY. Sentinel lymph node biopsy in endometrial cancer: meta-analysis of 26 studies. Gynecol Oncol. 2011;123:522-7.

29. Rossi EC, Kowalski LD, Scalici J, Cantrell L, Schuler K, Hanna RK, Method M, Ade M, Ivanova A, Boggess JF. A comparison of sentinel lymph node biopsy to lymphadenectomy for endometrial cancer staging (FIRES trial): a multicentre, prospective, cohort study. Lancet Oncol. 2017;18:384-92.
30. Bodurtha Smith AJ, Fader AN, Tanner EJ. Sentinel lymph node assessment in endometrial cancer: a systematic review and meta-analysis. Am J Obstet Gynecol. 2017;216:459-476 e410.

31. Darin MC, Gomez-Hidalgo NR, Westin SN, Soliman PT, Escobar PF, Frumovitz M, Ramirez PT. Role of indocyanine green in sentinel node mapping in gynecologic cancer: is fluorescence imaging the new standard? J Minim Invasive Gynecol. 2016;23:186-93.

32. Papadia A, Zapardiel I, Bussi B, Ghezzi F, Ceccaroni M, De Ponti E, Elisei F, Imboden S, de la Noval BD, Gasparri ML, et al. Sentinel lymph node mapping in patients with stage I endometrial carcinoma: a focus on bilateral mapping identification by comparing radiotracer Tc99m with blue dye versus indocyanine green fluorescent dye. J Cancer Res Clin Oncol. 2017;143:475-80.

33. Eriksson AG, Montovano M, Beavis A, Soslow RA, Zhou Q, Abu-Rustum NR, Gardner GJ, Zivanovic O, Barakat RR, Brown CL, et al. Impact of obesity on sentinel lymph node mapping in patients with newly diagnosed uterine cancer undergoing robotic surgery. Ann Surg Oncol. 2016;23:2522-8.

34. Todo Y, Kato H, Kaneuchi M, Watari H, Takeda M, Sakuragi N. Survival effect of para-aortic lymphadenectomy in endometrial cancer (SEPAL study): a retrospective cohort analysis. Lancet. 2010;375:1165-72.

35. Abu-Rustum NR, Chi DS, Leitao M, Oke EA, Hensley ML, Alektiar KM, Barakat $\mathrm{RR}$. What is the incidence of isolated paraaortic nodal recurrence in grade 1 endometrial carcinoma? Gynecol Oncol. 2008;111:46-8.

36. Abu-Rustum NR, Gomez JD, Alektiar KM, Soslow RA, Hensley ML, Leitao MM Jr, Gardner GJ, Sonoda Y, Chi DS, Barakat RR. The incidence of isolated paraaortic nodal metastasis in surgically staged endometrial cancer patients with negative pelvic lymph nodes. Gynecol Oncol. 2009;115:236-8.

37. Chiang AJ, Yu KJ, Chao KC, Teng NN. The incidence of isolated para-aortic nodal metastasis in completely staged endometrial cancer patients. Gynecol Oncol. 2011:121:122-5.

38. Khoury-Collado F, Murray MP, Hensley ML, Sonoda Y, Alektiar KM, Levine DA, Leitao MM, Chi DS, Barakat RR, Abu-Rustum NR. Sentinel lymph node mapping for endometrial cancer improves the detection of metastatic disease to regional lymph nodes. Gynecol Oncol. 2011;122:251-4.

39. Niikura H, Okamoto S, Yoshinaga K, Nagase S, Takano T, Ito K, Yaegashi N. Detection of micrometastases in the sentinel lymph nodes of patients with endometrial cancer. Gynecol Oncol. 2007;105:683-6.

40. Holloway RW, Gupta S, Stavitzski NM, Zhu X, Takimoto EL, Gubbi A, Bigsby GE, Brudie LA, Kendrick JE, Ahmad S. Sentinel lymph node mapping with staging lymphadenectomy for patients with endometrial cancer increases the detection of metastasis. Gynecol Oncol. 2016;141:206-10.

41. Cormier B, Rozenholc AT, Gotlieb W, Plante M, Giede C. Sentinel lymph node procedure in endometrial cancer: a systematic review and proposal for standardization of future research. Gynecol Oncol. 2015;138:478-85.

42. St Clair CM, Eriksson AG, Ducie JA, Jewell EL, Alektiar KM, Hensley ML, Soslow RA, Abu-Rustum NR, Leitao MM Jr. Low-volume lymph node metastasis discovered during sentinel lymph node mapping for endometria carcinoma. Ann Surg Oncol. 2016:23:1653-9.

43. Bezu C, Coutant C, Ballester M, Feron JG, Rouzier R, Uzan S, Darai E. Ultrastaging of lymph node in uterine cancers. J Exp Clin Cancer Res. 2010; 29:5.

44. Holloway RW, Abu-Rustum NR, Backes FJ, Boggess JF, Gotlieb WH, Jeffrey Lowery W, Rossi EC, Tanner EJ, Wolsky RJ. Sentinel lymph node mapping and staging in endometrial cancer: a society of gynecologic oncology literature review with consensus recommendations. Gynecol Oncol. 2017; 146(2):405-15. https://doi.org/10.1016/j.ygyno.2017.05.027. Epub 2017 May 28.

45. Zeimet AG, Reimer D, Huszar M, Winterhoff B, Puistola U, Azim SA, MullerHolzner E, Ben-Arie A, van Kempen LC, Petru E, et al. L1CAM in early-stage type I endometrial cancer: results of a large multicenter evaluation. J Natl Cancer Inst. 2013;105:1142-50.

46. Talhouk A, McConechy MK, Leung S, Yang W, Lum A, Senz J, Boyd N, Pike J, Anglesio M, Kwon JS, et al. Confirmation of ProMisE: a simple, genomicsbased clinical classifier for endometrial cancer. Cancer. 2017;123:802-13.

47. Cancer Genome Atlas Research N, Kandoth C, Schultz N, Cherniack AD, Akbani R, Liu Y, Shen H, Robertson AG, Pashtan I, Shen R, et al. Integrated genomic characterization of endometrial carcinoma. Nature. 2013;497:67-73. 